Antonio Ramos Archibold ${ }^{\mathrm{a}, \mathrm{b}, \mathrm{f}}$

3

\title{
Multi-mode heat transfer analysis during freezing of an encapsulated storage medium
}

4 5
Elias K. Stefanakos ${ }^{\mathrm{a}, \mathrm{d}}$

8

\author{
${ }^{a}$ Clean Energy Research Center, University of South Florida, Tampa, Fl, USA \\ ${ }^{b}$ Department of Mechanical Engineering, University of South Florida, Tampa, Fl, USA \\ ${ }^{c}$ Department of Chemical \& Biomedical Engineering, University of South Florida, Tampa, Fl, USA \\ ${ }^{d}$ Department of Electrical Engineering, University of South Florida, Tampa, Fl, USA \\ ${ }^{e}$ Department of Mechanical Engineering, Wichita State University, Wichita, KS, USA \\ ${ }^{f}$ Department of Mechanical Engineering, Universidad Autónoma del Caribe, Barranquilla, Colombia
}

${ }^{*}$ Corresponding author: Tel.: +1 813974 0956; fax: +1 813974 5250, e-mail address: goswami@ usf.edu 


\section{Multi-mode heat transfer analysis during freezing of an encapsulated storage medium}
Antonio Ramos Archibold ${ }^{\mathrm{a}, \mathrm{b}, \mathrm{f}}$
D. Yogi Goswami ${ }^{\mathrm{a}, \mathrm{c}, \dagger}$
Muhammad M. Rahman ${ }^{\mathrm{e}}$
Elias K. Stefanakos ${ }^{\mathrm{a}, \mathrm{d}}$ .

.

(1)

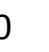

\section{Abstract}

${ }^{a}$ Clean Energy Research Center, University of South Florida, Tampa, Fl, USA

${ }^{b}$ Department of Mechanical Engineering, University of South Florida, Tampa, Fl, USA

${ }^{c}$ Department of Chemical \& Biomedical Engineering, University of South Florida, Tampa, Fl, USA

${ }^{d}$ Department of Electrical Engineering, University of South Florida, Tampa, Fl, USA

${ }^{e}$ Department of Mechanical Engineering, Wichita State University, Wichita, KS, USA

${ }^{f}$ Department of Mechanical Engineering, Universidad Autónoma del Caribe, Barranquilla, Colombia 


\section{Introduction}

Assessment of the fundamental thermal energy transport mechanisms during phase change in materials at relatively high operating temperatures has recently gained considerable attention in the solar energy research community because the urgent need to explore and develop advanced energy storage systems $[1,2,3]$ based on latent heat storage in solar thermal power plants. Special attention has been

42 devoted to the melting and freezing of thermal storage media confined in spherical or cylindrical 43 containers to be used in packed bed and shell and tube heat exchangers respectively $[4,5]$. The ability to 44 quantify and appropriately predict, from a theoretical perspective, the energy contributions from 45 conduction, convection and thermal radiation and their influence on the melting and freezing is necessary 46 to evaluate the thermal performance and obtain accurate thermal designs of the aforementioned systems $47[6,7,8,9]$.

An extensive literature review of the reported solutions of the PCM solidification problem reveals that the most commonly analyzed model assumes that the medium is initially at the phase change temperature. Therefore, no heat transfer is allowed across the liquid phase, and the heat only flows in the solid portion. This simplified model is known as the single phase Stefan problem. Closed-form, semianalytical solutions of the aforementioned problem has been presented by $[10,11,12,13,14,15,16,17]$ based on the similarity variable approach, asymptotic theory and parameter-perturbation methods. Tao [18] reported a numerical solution of the single phase Stefan problem. The formulation in which the presence of temperature gradients across the liquid phase is considered has been termed the two phase

56 Stefan problem and has been experimentally and numerically analyzed, during freezing in vertical tubes,

57 by Sparrow and Broadbent [19] and Sparrow and Ohkubo [20] respectively. The studies conclude that 58 initial superheating of the liquid moderately diminishes the frozen mass and the associated latent energy 59 extraction at small times, but has a small effect on these quantities at large times. Also, good agreement 60 was found between the numerical predictions and the experimental results. model, the initial temperature of the system was above the phase change temperature. However, only heat 
conduction was taken into account through the liquid phase. The study concludes that for small Stefan number conditions, the temperature within the inner liquid phase rapidly decreases to the phase change temperature. Consequently, heat conduction within the liquid does not affect the solidification process. Ismail and Henriquez [22] performed a parametric analysis on the single phase Stefan problem for water confined in a spherical shell. They discussed the influence of the capsule size, shell material, shell thickness and outer surface boundary condition on the PCM solidification rate. The study concludes that an increase of the Biot number at the external surface of the shell decreases the solidification time. Although the domain geometry and the boundary conditions are the same in the present analysis and the study reported in [22], the present analysis expands the previous one by including the contributions of thermal radiation and natural convection within the PCM on the net energy transport. These contributions were included in the equation of energy conservation and present a more realistic physical description of the problem.

Freezing experiments of n-hexadecane encapsulated in spherical shells have been reported by Chan and Tan [23]. The evolution of the solidification phase via visualization front is presented for different outer surface and initial temperatures. The study concludes that the solidification phase front progresses concentrically inwards from the colder outer surface of the sphere. Regin et al. [24] presented a numerical analysis on the solidification process of paraffin wax within a horizontal cylindrical capsule externally heated by a convection boundary condition.

The influence of the PCM volume reduction during the solidification in a partially filled spherical shell has been studied by Assis et al. [25]. In the model, the domain was initially partially filled with liquid paraffin wax, with air in the remaining volume. Numerical results and experimental observations show that an upper void space is created inside the capsule due to the PCM volume reduction during the solidification process. The study also concludes that natural convection heat transfer in the liquid phase is negligible for Stefan numbers below 1. Diffusion-controlled freezing of a micro encapsulated PCM has been analyzed by Yang and Zhao [26]. The influence of thermal radiation on the single phase Stefan problem for non-opaque media have been largely limited to planar systems [27, 28, 29, 30, 31, 32] while 
the problem in spherical media has received little attention; especially when all the fundamental heat transfer modes are simultaneously present.

The objective of the present work is to examine the influence of simultaneous conduction, convection and thermal radiation on the freezing dynamics of a non-opaque media, with spectral dependence on its optical properties, encapsulated in a closed spherical shell at relatively high temperatures, in order to estimate the induced error in the solutions where participating thermal radiation within the PCM has been omitted. To accomplish that, a two-dimensional, axisymmetric, transient model has been solved numerically. The primary motivation of this investigation is to provide additional information about the key elements in any phase change problem, i.e., solid-liquid interface position and phase distribution at a given time after the application of a thermal driving force. Particularly under operating thermal conditions related to thermal energy storage for concentrating solar power plants.

\section{Heat transfer analysis}

The considered system is schematically shown in a cross sectional view in Figure 1. It consists of an opaque, gray and a diffuse spherical shell of inner radius $R_{i}$ and wall thickness $\delta$. Initially the shell inner volume is completely filled with liquid PCM at temperature $T_{o}$, which is higher than the storage material melting temperature $T_{m}$. At time $t>0$, the outer boundary surface, whose emissivity was set to unity, is subjected to a convective boundary condition characterized by a heat transfer coefficient $h_{\infty}$ and a free stream temperature $T_{\infty}$, which is lower than $T_{m}$. The PCM is treated as a semitransparent, non-gray, medium being emitting and absorbing. Uniform and equal index of refraction of each phase has been assumed. The scattering of thermal radiation has been neglected in this study because of the lack of the PCM property data.

Multiple physical assumptions have been considered in the mathematical model and attention will now be turned to discuss their applicability. Experimental observations on the freezing of a low temperature PCM [23] and the natural convection heat transfer to a fluid contained in spherical containers [33] suggest that both the phase change process and the buoyancy induced fluid motion can be modeled 
as axisymmetric. It was also reported by Chow and Akins [33] that for Rayleigh numbers below $10^{7}$, laminar flow was found to exist. In the present study the range of the examined Rayleigh numbers was $7.92 \times 10^{4} \leq R a \leq 2.0 \times 10^{7}$. Therefore the process has been modeled as axisymmetric and laminar flow has been assumed for the fluid motion within the liquid phase. In order to account for the natural convection, temperature dependent density within the liquid phase has been defined only in the body force terms of the momentum equations based on the Boussinesq approximation.

The PCM is assumed to be a continuous, homogeneous and isotropic substance and its thermophysical properties have been taken from [34]. The melting of $\mathrm{NaNO}_{3}$ takes place in the interval between $306.3^{\circ} \mathrm{C}$ and $306.8^{\circ} \mathrm{C}$ and the wall thickness used in all the study cases was $1 \mathrm{~mm}$. The equations that the governing equations may be written as:

127

$$
\frac{\partial \rho}{\partial t}+\frac{1}{r^{2}} \frac{\partial}{\partial r}\left(\rho \vartheta_{r} r^{2}\right)+\frac{1}{r \sin \theta} \frac{\partial}{\partial \theta}\left(\rho \vartheta_{\theta} \sin \theta\right)=0
$$

128

$$
\rho\left(\frac{\partial \vartheta_{r}}{\partial t}+v_{r} \frac{\partial \vartheta_{r}}{\partial r}+\frac{\vartheta_{\theta}}{r} \frac{\partial \vartheta_{r}}{\partial \theta}-\frac{\vartheta_{\theta}^{2}}{r}\right)=-\frac{\partial P}{\partial r}+\eta\left[\nabla^{2} \vartheta_{r}-\frac{2 \vartheta_{r}}{r^{2}}-\frac{2}{r^{2} \sin \theta} \frac{\partial\left(\vartheta_{\theta} \sin \theta\right)}{\partial \theta}-\frac{2 \vartheta_{\theta} \cot \theta}{r^{2}}\right]-\rho g_{r} \beta\left(T-T_{m}\right)
$$

129

$$
\rho\left(\frac{\partial \vartheta_{\theta}}{\partial t}+\vartheta_{r} \frac{\partial \vartheta_{\theta}}{\partial r}+\frac{\vartheta_{\theta}}{r} \frac{\partial \vartheta_{\theta}}{\partial \theta}+\frac{\vartheta_{r} \vartheta_{\theta}}{r}\right)=-\frac{1}{r} \frac{\partial P}{\partial r}+\eta\left[\nabla^{2} \vartheta_{\theta}+\frac{2}{r^{2}} \frac{\partial \vartheta_{r}}{\partial \theta}-\frac{\vartheta_{\theta}}{r^{2} \sin ^{2} \theta}\right]-\rho g_{\theta} \beta\left(T-T_{m}\right)
$$

130

$$
\frac{\partial h}{\partial t}+\frac{1}{r^{2}} \frac{\partial}{\partial r}\left(r^{2} \vartheta_{r} h\right)+\frac{1}{r \sin \theta} \frac{\partial}{\partial \theta}\left(\vartheta_{\theta} \sin \theta h\right)=\alpha \nabla^{2} h-\frac{1}{\rho c_{p}}\left(\frac{\partial \lambda}{\partial t}+\frac{1}{r^{2}} \frac{\partial}{\partial r}\left(r^{2} \vartheta_{r} \lambda\right)+\frac{1}{r \sin \theta} \frac{\partial}{\partial \theta}\left(\vartheta_{\theta} \sin \theta \lambda\right)\right)-\nabla \cdot q_{r}
$$

The last term on the right-hand side of the energy equation accounts for the thermal radiation contribution, and it is coupled with the radiation intensity via the equation of radiative transfer (RTE). For

134 diffuse radiation and isotropic scattering in a spherically symmetric medium the RTE [35] can be written 135 as: 


$$
\frac{\mu}{r^{2}} \frac{\partial\left[r^{2} I(r, \mu)\right]}{\partial r}+\frac{1}{r} \frac{\partial\left[\left(1-\mu^{2}\right) I(r, \mu)\right]}{\partial \mu}+\kappa_{a} I(r, \mu)=\kappa_{a} I_{b}(T)
$$

where $I(r, \mu)$ is the radiation intensity, which is a function of the position and direction, $\mu$ is the cosine of the angle between the direction $\Omega$ of the beam and the extension of the radius vector $r, I_{b}(T)$ is the intensity of a black body radiation at the temperature of the medium. In the discrete ordinate method, the RTE is approximated by discretizing the entire incident solid angle using a finite number of ordinate directions [36, 37, 38, 39]. Therefore, the equation [5] is written for each ordinate and reduces to a discrete system of differential equations, sufficient to solve for radiation intensity $\left(I_{i}\right)$ at every location within the medium. The set of the equation results in:

$$
\frac{\mu_{i}}{r^{2}} \frac{\partial\left[r^{2} I_{i}\right]}{\partial r}+\frac{1}{r} \frac{\partial\left[\left(1-\mu^{2}\right) I\right]_{\mu=\mu_{i}}}{\partial \mu}+\kappa_{a} I_{i}=\kappa_{a} I_{b}
$$

where the subscript i denotes the ordinate directions. Once the radiation intensity is known, the local radiative heat flux $\left(\mathrm{q}_{\mathrm{r}}\right)$ can be determined based on the following expression:

$$
q_{r}=2 \pi \int_{-1}^{1} I(r, \mu) \mu d \mu=2 \pi \sum_{i=1}^{n} \mu_{i} w_{i} I_{i}
$$

The control volume technique, which employs an implicit scheme based on the SIMPLE (Semi-Implicit Method for Pressure-Linked Equations) algorithm [40], has been used to solve the governing equations. The computational domain has been discretized based on a structured grid consisting of 5580 quadrilateral cells with refined mesh distribution in the vicinity of the inner wall of the shell. Commercially available software Ansys/Fluent v12 was employed for the calculations. The details of the employed numerical procedure have been described in reference [41].

Two different properties have been selected to evaluate the effect of the mesh grid size on the solution of the model. The mass average solid fraction and the dimensionless temperature $\left(\Theta_{n}\right)$ of a node located at the horizontal axis of the domain have been plotted as a function of the dimensionless time (as seen in Figure 2) for different grid densities on the study case with $R_{i}=15 \mathrm{~mm}, T_{o}-T_{m}=20^{\circ} \mathrm{C}$, and $T_{m}-T_{\infty}=20.5^{\circ} \mathrm{C}$. It may be seen from Figure 2 that for the given conditions, additional refining of the grid from 1400 to 10400 elements has no influence on the freezing rate. No significant difference is observed in the solidification dynamics with the freezing time approximately to 28.33 minutes. The same trend is observed in the dimensionless temperature response during the liquid phase sensible cooling and phase transition process $0 \leq t \leq 13.1 \mathrm{~min}$. However, some minor differences are observed at the beginning of the solid phase sensible cooling process with slightly faster temperature response 
with the increase of the element size. A grid distribution with 5580 elements, where the minimum and maximum element sizes are $0.29 \times 0.15 \mathrm{~mm}$ and $0.15 \times 0.55 \mathrm{~mm}$ (width $\mathrm{x}$ height) respectively, was found sufficient to achieve independence of the solution.

The time step $\left(t_{s p}\right)$ used in the present study was $0.008 \mathrm{sec}$ and it was selected based on the results of the time step independent analysis reported by the authors at [34] for a similar problem. In the range of $0.002 \leq t_{s p} \leq$ $0.009 \mathrm{~s}$, It was concluded that the solution is independent of the time step. The proposed numerical model has been validated against the semi-analytical solution of the single phase Stefan problem, in the absence of thermal radiation, reported by Hill and Kucera [16]. A comparison is provided in Figure 3 which illustrates the variation of the dimensionless solid/liquid interface with respect to the dimensionless time for parametric values of the Stefan and Biot number equal to 0.5 and 1 respectively. Reasonably good agreement is observed between the numerical and the analytical results. However, as it was mentioned in the introduction section, the proposed model also incorporates the participating thermal radiation within the PCM during the phase change process, which is missing in the simplified Stefan problem where the heat of fusion is assumed to be removed by conduction only. Therefore a second validation test has been performed in order to verify the thermal response of the one dimensional transient solidification problem of a semitransparent planar medium in the presence of simultaneous conduction and thermal radiation. Figure 4 shows the solid fraction distribution along the characteristic length of the slab for different dimensionless time $(\xi)$. The figure also includes a comparison between the results reported by Parida et al. [42] and the predictions obtained from our simulation of the same problem. The general trends for both simulations are the formation of a mushy zone that extends into the slab early in the process and the propagation of the solid phase into the slab as the freezing proceeds. Reasonably good agreement is observed.

\section{Results and discussion}

The parameters analyzed during the course of this investigation are the melt degree of superheat $\left(T_{o}-T_{m}\right)$, the external heat transfer coefficient that characterizes the outer shell wall boundary condition, the size of the shell and the participating thermal radiation within the PCM. These parameters have been selected based on a practical point of view of the possible use of $\mathrm{NaNO}_{3}$ for storage of thermal energy in concentrating solar power plants. Eight different study cases have been defined to identify the 
aforementioned parameters and are presented in table 1 . Under the present conditions, the liquid phase degree of superheat will activate the natural convection energy transport and consequently affect the freezing dynamic of the PCM, at least during the early periods of the process, as reported by Sparrow and Broadbent [19] for the solidification in vertical cylinders. Four superheating conditions were considered, $1,5,10$ and $20^{\circ} \mathrm{C}$ above the PCM melting temperature respectively. Study cases $1-3$ and 5 represent this effect. As it was mentioned before, a uniform convective thermal boundary condition was imposed at the outer wall of the capsule in order to represent the discharging process in a packed bed system. heat transfer coefficients $\left(h_{\infty}\right)$ have been evaluated and their influence on the thermal behavior of the system was analyzed. Study cases 4-6 describe this effect. Three different shell sizes have been analyzed in this study with inner radius of 15,25 and $35 \mathrm{~mm}$ respectively. These conditions are represented by study cases 6-8. In order to account for the participating thermal radiation, the PCM has been modeled as a semitransparent, non-gray medium in all the study cases presented in table 1. A seven-band model, based on the experimental results reported by Ramdas [43] has been used to account for the spectral dependence of the PCM optical properties. A limiting case in which the PCM has been treated as a nonattenuating and non-emitting medium with shell inner surface emissivity equal to zero has been also included in the analysis, in order to provide a reference condition in which the thermal radiation does not the same as the used in the study case 5. Further, in this section a discussion about the relevant radiant properties is also included.

Predictions of the evolution of the solidification process at various time instants are presented in

207 Figure 5. Each plot is composed of two separate halves divided by the symmetry line of the domain which 208 is used as the ordinate in the defined radial and axial $(\mathrm{Z})$ coordinate system. Isotherms are presented in the 209 left half while the streamline contours and solid fraction distribution are shown in the right half. central core of the capsule upward. The ascending fluid transfers its heat to the adjacent cooler fluid near 
the inside wall. It is cooled down and descends nearby the inner shell wall. Hence an unsteady, clockwise circulating flow is formed in the melt layer which is characterized by the closed streamline maps observed on the right side of Figures $5 \mathrm{a}$ and $5 \mathrm{~b}$. This recirculation pattern delivers relatively hot liquid to the upper region of the capsule, where its presence retards the cooling process.

During this short time scale (relative to the period to complete the PCM freezing), the hot fluid 217 continuously arrives into this region, impinges on the inner wall and displaces the cooler fluid down. 218 Inspection of the temperature contours of Figures 5a and 5b reveals a stratified temperature field with the 219 highest temperature in the upper zone. During its travel along the inner wall, the displaced fluid is being 220 cooled by the outer wall and descends toward the lower portion of the capsule where the temperature 221 drops below the melting point and therefore inward solidification begins. A closer view of the right hand 222 side of Figures 5a and 5b illustrates the formation of the first PCM solid layer at the bottom of the shell. 223 During this time scale the buoyancy induced flow pattern in the melt layer is characterized by a thin layer of liquid PCM flowing downward and a large core of fluid moving upward. The thickness of the downflow fell in the range of 12 to $18.7 \%$ of the inner radius of the shell at early times. These findings are in agreement with the experimental observations of the natural convection flow inside spherical enclosures reported by Chow and Akins [33]. freezing $(0 \leq t \leq 150 s)$ where natural convection is believed to be the dominant energy transport mechanism, the temperature profile at the horizontal radius is presented in Figure 6 for different times. Sharp temperature gradients are developed in the fluid adjacent to the inner wall while uniform 232 temperature is observed across the thickness of the PCM liquid layer. This trend is associated with the 233 formation of the thermal boundary layer near the inner shell wall. Similar results were reported by 234 Sparrow et al. [44] for the melting problem in vertical cylinders. Inspection of the left hand side of Figure 5c shows that the temperature gradient within the melt 236 layer that was responsible for the natural convection motion is no longer in evidence, therefore, a 237 significant reduction of the streamline contour intensity is also observed as compared with the previous 
time instant. Another important observation of Figure $5 \mathrm{c}$ is that the temperature within the melt layer is slightly above the phase change temperature, meaning that during the first 240 s of the process the

temperature has dropped approximately $20^{\circ} \mathrm{C}$. With the absence of the hot flow at the top of the shell the inward solidification proceeds and solid PCM is observed in the vicinity of all the inner shell.

Figures $5 \mathrm{~d}$ to $5 \mathrm{i}$ illustrate the freezing dynamics of the PCM with almost concentric ring shape for the isotherms as well as the solid/liquid interface. The absence of the temperature gradients within the melt layer is the reason why the streamlines contours are not included in figures $5 \mathrm{~d}$ to $5 \mathrm{i}$.

To place these findings in perspective, the events that occur during the presented freezing process can be divided into two different time scales. A short time scale $0 \leq t \leq 150 s$ in which natural convection plays a first order role, but rapidly terminated because of the sharp decrease of the liquid core temperature. On the other hand, a second and larger time scale (relative to the period to complete the PCM freezing) that shows solid-phase isotherms close to the solution of the Laplace equation, where it is assumed that the leading energy transport mechanisms are conduction and radiation. In that context the simultaneous interaction between the conductive and the radiative transfer provides a more effective mechanism to transport the liberated latent heat of fusion from the solid/liquid interface to the outer shell wall.

It has been previously reported [45] that the presence of thermal radiation significantly improves the heating or cooling rates as compared with the pure conduction systems. Based on that, a spectral absorption coefficient has been defined for both solid and liquid PCM phases in order to account for the participating thermal radiation that may occur during the freezing process. Figure 7 shows the experimental measurements of the near infrared transmittance of $\mathrm{NaNO}_{3}$, reported by Ramdas [43] and the approximate seven band model that was used during the calculation process. For each band, a uniform absorption coefficient has been estimated while the contribution of scattering to the extinction of thermal radiation has been neglected. 

difficulties are anticipated to analyze other PCMs in the presented model. For instance, the influence of thermal radiation on the melting of $\mathrm{NaCl}$ with enhanced radiative properties was reported by [46].

Recent experimental contributions on the radiative properties of high-temperature eutectic systems reported by Myers et al. [47] can be utilized to extend and compare the results reported in this

267 investigation. The predicted variation of the solid fraction with time for the limiting case and the study 268 case 5 is presented in Figure 8. It may be seen that faster solidification is achieved when thermal radiation 269 is considered. The solidification time decreases $17 \%$ when thermal radiation is included in the model. A closer look of the solid fraction curves reveals that during the early times, the solid fraction is equal to zero, indicating the existence of the pure natural convection motions within the liquid PCM.

As the freezing initiates, a small difference is observed between the curves with study case 5 273 predicting a slightly faster solidification. As the solid layer increases in size, the contribution of thermal 274 radiation becomes more significant in the presence of conduction, resulting in a more pronounced 275 difference. In the absence of radiation heat transfer, the rate of heat transfer decreases as the solid layer thickness increases, since conduction is the only mode of heat transfer through the solid PCM. However, 277 the presence of radiation allows the heat transfer from the inner liquid core to the shell walls through the 278 solid layer attenuated only by the absorption and scattering by the solid PCM layer. Consequently, radiant 279 heat transfer increases the cooling rate from the liquid core to the outer surface of the shell, resulting in a faster freezing process.

The predicted energy transfer interactions at the inner shell wall are also included in Figure 8 and 282 are displayed in broken lines. In order to appropriately correlate the heat transfer data, two different 283 dimensionless parameters have been defined, the Nusselt number $(\mathrm{Nu})$ which is estimated based on the 284 conductive and the convective heat fluxes $\left(q_{c d}\right.$ and $\left.q_{c v}\right)$ and the dimensionless radiative flux $(\Psi)$ which 285 has been calculated based on the radiation heat flux $\left(q_{r}\right)$. It should be noticed that the limiting case is only 286 characterized by the Nusselt number because of the absence of thermal radiation while the case 5 287 accounts for all the energy transport modes. Both curves are multiplied by the Stefan number in order to 
accommodate them in the solid fraction plot. A sharp decrease is initially observed in the heat transfer curves during the pure natural convection period. A change in slope in both of the curves is found to be coincident with the starting of the freezing process as may be seen from the solid fraction curves. As expected the contribution of thermal radiation enhances the energy interaction at the inner shell wall.

In order to provide a quantitative notion of the velocity magnitudes and the flow patterns developed in the melt layer during the early periods of the process, the axial velocity distribution along the horizontal radius of the capsule at different times is presented in Figure 9. The flow structure can be described as follows. A thin layer of fluid flows downward in the vicinity of the inner shell wall, where the maximum velocity is attained, and an upward flow characterizes the fluid in the center of the capsule. As the driving temperature difference within the melt layer decreases with time (see Fig. 6) the natural convection flow loses its strength and consequently the downward and inward velocities also decrease. A closer view of Figure 9 reveals that the thickness of both flows increases as the process proceeds. At 180s, the velocity magnitude was found to be significantly smaller as compared to the values at $60 \mathrm{~s}$.

The effects of the initial superheating temperature on the solid fraction and the dimensionless energy transfer at the inner shell wall are presented in Figure 10. It may be seen from the bottom of the solid fraction curves that the larger the degree of superheating the longer to start the freezing. Even though, the liquid superheat clearly affects the freezing dynamics early in the process. Once the energy transfer from the liquid phase to the solid/liquid interface has ceased, the remaining energy transport mechanisms are radiation and conduction within the solid phase which essentially remain unchanged under the analyzed thermal conditions of cases 1-3 and 5. This fact could be responsible for the small differences observed in all of the curves at the latest stages of the process.

The influence of the external heat transfer coefficient on the solid fraction rate is depicted in Figure 11. The heat transfer coefficient values of $45.4,226.7$ and $453.5 \mathrm{~W} / \mathrm{m}^{2} \mathrm{~K}$ have been evaluated which correspond to Biot numbers equal to 1,5 and 10 respectively. As expected a reduction in the solidification time was found with the increase of the Biot number. However the time reduction was more pronounced in the case where $\mathrm{Bi}=1$ as compared with the study cases where the Biot number was 5 and 
10. No attempt to analyze the influence of the free stream temperature on the thermal dynamics of the process has been made in this investigation. The reason is because the trend that characterizes the subcooling degree is fully expected and has been previously discussed.

The effect of the shell size on the solidification rate is presented in Figure 12. Three different shell sizes have been analyzed with inner radius varying from 15 to $35 \mathrm{~mm}$. It may be observed that the smaller the capsule the faster the solidification. As shown in Figure 13, the predicted solid fraction for all the study cases, except for the study case 4, has been plotted as a function of a dimensionless group $\left(F o S t e_{L}^{a} G r_{R}^{b} B i^{c}\right)$ that takes into consideration the controlling parameters analyzed in this investigation. The following equation is proposed to be used to correlate the solid fraction of the analyzed cases:

$$
S F=1.017 \operatorname{erf}\left[0.1\left(F o S t e_{L}^{a} G r_{R}^{b} B i^{c}\right)^{1.04}\right]
$$

It was found that, for the exponents $a=-0.18, b=0.13, c=0.31$, all the data merged together in a single line. A small deviation from the proposed correlation is observed in the early stages of the process in study cases 5, 6 and 7 and it is related to the wide range considered for the Biot number, which controls range of the controlling parameters:

$$
\begin{gathered}
0.0096 \leq \text { Ste }_{L} \leq 0.195 \\
8.77 \times 10^{3} \leq G r_{R} \leq 2.42 \times 10^{6} \\
5 \leq B i \leq 23.34 \\
P r \cong 9.0
\end{gathered}
$$

\section{Summary and conclusions}

The numerical solution of the solidification problem of a semitransparent, non-gray PCM confined in a spherical shell has been presented. In contrast to the well-known conduction-dominated model of the single phase Stefan problem, the present study has taken into consideration the energy contributions due to simultaneous convection, radiation and conduction during the solidification at relatively high temperature. The discrete ordinate method was used to solve the equation of radiative transfer, and the finite volume scheme was used to solve the equations for mass, momentum and energy 
- In the presence of all the fundamental heat transfer mechanisms, it was found that the contribution of thermal radiation on the solidification process of $\mathrm{NaNO}_{3}$ is to reduce the solidification time by $17 \%$ as compared with the limiting case where thermal radiation has been neglected.

- During the early periods of the process, an increase of the initial liquid-phase superheating degree was found to accelerate the freezing. Once the natural convection which is induced by the temperature differences within the liquid phase is terminated, the remaining energy transport mechanisms take control of the process until the final stages, attenuating in this way, the initial effect of the natural convection. It was also shown that the presence of natural convection induces the initial formation of an asymmetrical solid PCM layer from the bottom of the capsule. It is believed that this factor is responsible for the slight variation in the solid layer thickness along the shell symmetry line, with the smallest layer thickness at the top and the greatest layer thickness at the bottom. the Biot number decreases from 10 to 5 .

- For a fixed thermal boundary condition, the solidification time decreases $60.92 \%$ and $21.23 \%$ as $R_{i}$ decreases from $35 \mathrm{~mm}$ to 15 and $25 \mathrm{~mm}$ respectively.

- An expression was obtained for the solid fraction as a function of the controlling parameters of the process. This can be readily used to estimate the PCM phase distribution at a given time when solving problems of latent heat thermal energy storage contained in a packed bed heat exchanger. 


\section{Acknowledgements}

361 The work presented herewith has been funded by the U.S. Department of Energy through the Advanced Research Projects Agency-Energy (ARPA-E) under the award number: DE-AR0000179. The authors also would like to acknowledge the use of the services provided by the Research Computing at the University of South Florida.

\section{References}

[1] H. Shabgard, C. W. Robak, T. L. Bergman and A. Faghri, "Heat transfer and exergy analysis of cascaded latent heat storage with gravity-assisted heat pipes for concentrating solar power applications," Solar Energy, vol. 86, pp. 816-830, 2012.

[2] J. F. Feldhoff, K. Schmitz, M. Eck, L. Schnatbaum-Laumann, D. Laing, F. Ortiz-Vives and J. Schulte-Fischedick, "Comparative system analysis of direct steam generation and synthetic oil parabolic trough power plants with integrated thermal storage," Solar Energy, vol. 86, pp. 520-530, 2012.

[3] S. Kuravi, J. Trahan, D. Y. Goswami, M. M. Rahman and E. K. Stefanakos, "Thermal energy storage technologies and systems for concentrating solar power plants," Progress in Energy and Combustion Science, vol. 39, pp. 285-319, 2013.

[4] H. Peng, H. Dong and X. Ling, "Thermal investigation of PCM-based high temperature thermal energy storage in packed bed," Energy Conversion and Management, vol. 81, pp. 420-427, 2014.

[5] K. Nithyanandam, R. Pitchumani and A. Mathur, "Analysis of a latent thermocline storage system with encapsulated phase change materials for concentrating solar power," Applied Energy, vol. 113, pp. 1446-1460, 2014.

[6] A. R. Archibold, A. Bhardwaj, M. M. Rahman, D. Y. Goswami and E. L. Stefanakos, "Thermal assessment of a latent leat energy storage module using a high temperature phase change material with enhanced radiative properties," in Proceedings of the ASME 2014 International Mechanical Engineering Congress \& Exposition, Nov. 14-20, Montreal, Canada, 2014.

[7] T. Alam, J. Dhau, D. Goswami, M. Rahman and E. Stefanakos, "Experimental investigation of a packed-bed latent heat thermal storage system with encapsulated phase change material," in Proceedings of the ASME 2014 International Mechanical Engineering Congress \& Exposition, Nov. 14-20, Montreal, Canada, 2010.

[8] A. R. Archibold, M. M. Rahman, D. Y. Goswami and E. L. Stefanakos, "High temperature latentheat thermal energy storage module with enhanced combined mode heat transfer," in Proceedings of the ASME 2014 International Mechanical Engineering Congress \& Exposition, Nov. 14-20, Montreal, Canada, 2014. 
[9] S. Bellan, J. Gonzalez-Aguilar, A. R. Archibold, M. Romero, M. M. Rahman, D. Yogi Goswami and E. K. Stefanakos, "Transient numerical analysis of storage tanks based on encapsulated PCMs for heat storage in concentrating solar power plants," in 2013 Ises Solar World Congress, Cancún, Mexico. Nov. 4-7, 2013.

[10] S. H. Cho and J. E. Sunderland, "Phase change of spherical bodies," International Journal of Heat and Mass Transfer, vol. 13, pp. 1231-1233, 1970.

[11] Y.-P. Shih and T.-C. Chou, "Analytical solutions for freezing a saturated liquid inside or outside spheres," Chemical Engineering Science, vol. 26, pp. 1787-1793, 1971.

[12] R. Pedroso and G. Domoto, "Inward spherical solidification- solution by the method of strained coordinates," International Journal of Heat and Mass Transfer, vol. 16, pp. 1037-1043, 1973.

[13] D. Riley, F. Smith and G. Poots, "The inward solidification of spheres and circular cylinders," International Journal of Heat and Mass Transfer, vol. 17, pp. 1507-1516, 1974.

[14] K. Stewartson and R. Waechter, "On Stefans problem for spheres," Proceedings of the Royal Society of London. A. Mathematical and Physical Sciences, vol. 348, pp. 415-426, 1976.

[15] A. Soward, "A unified approach to Stefans problem for spheres and cylinders," Proceedings of the Royal Society of London. A. Mathematical and Physical Sciences, vol. 373, pp. 131-147, 1980.

[16] J. M. Hill and A. Kucera, "Freezing a saturated liquid inside a sphere," International journal of heat and mass transfer, vol. 26, pp. 1631-1637, 1983.

[17] M. Prud'homme, D. L. Nguyen and T. H. Nguyen, "A heat transfer analysis for solidification of slabs, cylinders, and spheres," Journal of Heat Transfer, vol. 111, pp. 699-705, 1989.

[18] L. C. Tao, "Generalized numerical solutions of freezing a saturated liquid in cylinders and spheres," AIChE Journal, vol. 13, pp. 165-169, 1967.

[19] E. Sparrow and J. Broadbent, "Freezing in a vertical tube," Journal of Heat Transfer, vol. 105, pp. 217-225, 1983.

[20] E. Sparrow and Y. Ohkubo, "Numerical predictions of freezing in a vertical tube," Numerical Heat Transfer, Part A: Applications, vol. 9, pp. 79-95, 1986.

[21] S. W. McCue, B. Wu and J. M. Hill, "Classical two-phase Stefan problem for spheres," Proceedings of the Royal Society A: Mathematical, Physical and Engineering Science, vol. 464, pp. 2055-2076, 2008.

[22] K. Ismail and J. Henríquez, "Solidification of PCM inside a spherical capsule," Energy conversion and management, vol. 41, pp. 173-187, 2000.

[23] C. Chan and F. Tan, "Solidification inside a sphere-an experimental study," International communications in heat and mass transfer, vol. 33, pp. 335-341, 2006. 
[24] A. Regin, S. Solanki and J. Saini, "Solidification of phase change material inside a cylindrical capsule," in Proceedings of 18th National and 7th ISHMT-ASME Heat and Mass Transfer Conference, Guwahati, India, 2006.

[25] E. Assis, G. Ziskind and R. Letan, "Numerical and experimental study of solidification in a spherical shell," Journal of Heat Transfer, vol. 131, p. 024502, 2009.

[26] J. Yang and C. Zhao, "Solidification analysis of a single particle with encapsulated phase change materials," Applied Thermal Engineering, vol. 51, pp. 338-346, 2013.

[27] M. Abrams and R. Viskanta, "The effects of radiative heat transfer upon the melting and solidification of semitransparent crystals," Journal of Heat Transfer, vol. 96, pp. 184-190, 1974.

[28] S. Chan, D. Cho and G. Kocamustafaogullari, "Melting and solidification with internal radiative transfer-a generalized phase change model," International Journal of Heat and Mass Transfer, vol. 26, pp. 621-633, 1983.

[29] S. Chan and K. Hsu, "The mushy zone in a phase change model of a semitransparent material with internal radiative transfer," Journal of heat transfer, vol. 110, pp. 260-263, 1988.

[30] C. Yao, B. Chung and G.-X. Wang, "Mushy zone equilibrium solidification of a semitransparent layer subject to radiative and convective cooling," International journal of heat and mass transfer, vol. 45, pp. 2397-2405, 2002.

[31] P. Lapka and P. Furmanski, "Numerical modelling of solidification processes of semitransparent materials using the enthalpy and the finite volume methods," Heat and Mass Transfer, vol. 44, pp. 937-957, 2008.

[32] H.-L. Yi, C.-H. Wang, H.-P. Tan and Y. Zhou, "Radiative heat transfer in semitransparent solidifying slab considering space-time dependent refractive index," International Journal of Heat and Mass Transfer, vol. 55, pp. 1724-1731, 2012.

[33] M. Chow and R. Akins, "Pseudosteady-state natural convection inside spheres," Journal of Heat Transfer, vol. 97, pp. 54-59, 1975.

[34] A. R. Archibold, J. Gonzalez-Aguilar, M. M. Rahman, D. Yogi Goswami, M. Romero and E. K. Stefanakos, "The melting process of storage materials with relatively high phase change temperatures in partially filled spherical shells," Applied Energy, vol. 116, pp. 243-252, 2014.

[35] S. Chandrasekhar, Radiative transfer, New York: Dover Publications, 1960.

[36] R. Viskanta and M. Mengüc, "Radiation heat transfer in combustion systems," Progress in Energy and Combustion Science, vol. 13, pp. 97-160, 1987.

[37] W. Fiveland, "Discrete-ordinates solutions of the radiative transport equation for rectangular enclosures," ASME Transactions Journal of Heat Transfer, vol. 106, pp. 699-706, 1984. 
[38] K. Sen and S. J. Wilson, Radiative Transfer in Curved Media: Basic and Mathematical Methods for Radiative Transfer and Transport Problems in Participating Media of Spherical and Cylindrical Geometry, World Scientific, 1990.

[39] R. Siegel and J. Howell, Thermal radiation heat transfer, Taylor \& Francis Group, 2002.

[40] S. V. Patankar, Numerical heat transfer and fluid flow, Taylor \& Francis Group, 1980.

[41] A. R. Archibold, M. M. Rahman, D. Y. Goswami and E. K. Stefanakos, "Analysis of heat transfer and fluid flow during melting inside a spherical container for thermal energy storage," Applied Thermal Engineering, vol. 64, pp. 396-407, 2014.

[42] P. Parida, R. Raj, A. Prasad and S. Mishra, "Solidification of a semitransparent planar layer subjected to radiative and convective cooling," Journal of Quantitative Spectroscopy and Radiative Transfer, vol. 107, pp. 226-235, 2007.

[43] A. Ramdas, "The infra-red absorption spectra of sodium nitrate and calcite," in Proceedings of the Indian Academy of Sciences, Vol. XXXVIII, No. 3, Sec. A, 1953.

[44] E. Sparrow, S. Patankar and S. Ramadhyani, "Analysis of melting in the presence of natural convection in the melt region," Journal of Heat Transfer, vol. 99, pp. 520-526, 1977.

[45] R. Viskanta and R. Merriam, "Heat transfer by combined conduction and radiation between concentric spheres separated by radiating medium," Journal of Heat Transfer, vol. 90, pp. 248-256, 1968.

[46] A. R. Archibold, M. M. Rahman, D. Yogi Goswami and E. K. Stefanakos, "The effect of radiative heat transfer during the melting process of a high temperature phase change material confined in a spherical shell," Applied Energy, vol. 138, pp. 675-684, 2015.

[47] P. Myers, D. Y. Goswami and E. K. Stefanakos, "Molten salt spectroscopy for quantification of radiative absorption in novel metal chloride-enhanced thermal storage media," in Proceedings of the ASME 2014 International Mechanical Engineering Congress \& Exposition, Nov. 14-20, Montreal, Canada, 2014.

\section{Nomenclature}

$c_{p} \quad$ specific heat at constant pressure $(\mathrm{J} / \mathrm{kg} \mathrm{K})$

Fo Fourier number $\left(\kappa / \rho \mathrm{c}_{\mathrm{p}}\right)\left(\mathrm{t} / \mathrm{R}_{\mathrm{i}}{ }^{2}\right)$

g gravitational acceleration $\left(\mathrm{m} / \mathrm{s}^{2}\right)$

$G r_{R} \quad$ Grashof number $\left(g \beta \Delta T R_{i}^{3} \rho^{2} / \eta^{2}\right)$

$h_{\infty} \quad$ external heat transfer coefficient $\left(\mathrm{W} / \mathrm{m}^{2} \mathrm{~K}\right)$

$h \quad$ sensible enthalpy $(\mathrm{J} / \mathrm{kg})$

$\mathrm{L} \quad$ Latent heat of fusion $(\mathrm{J} / \mathrm{kg})$

$\mathrm{Nu} \quad$ Nusselt number $\left(q_{c d}+q_{c v}\right) R_{i} / \kappa\left(T_{m}-T_{\infty}\right)$ 


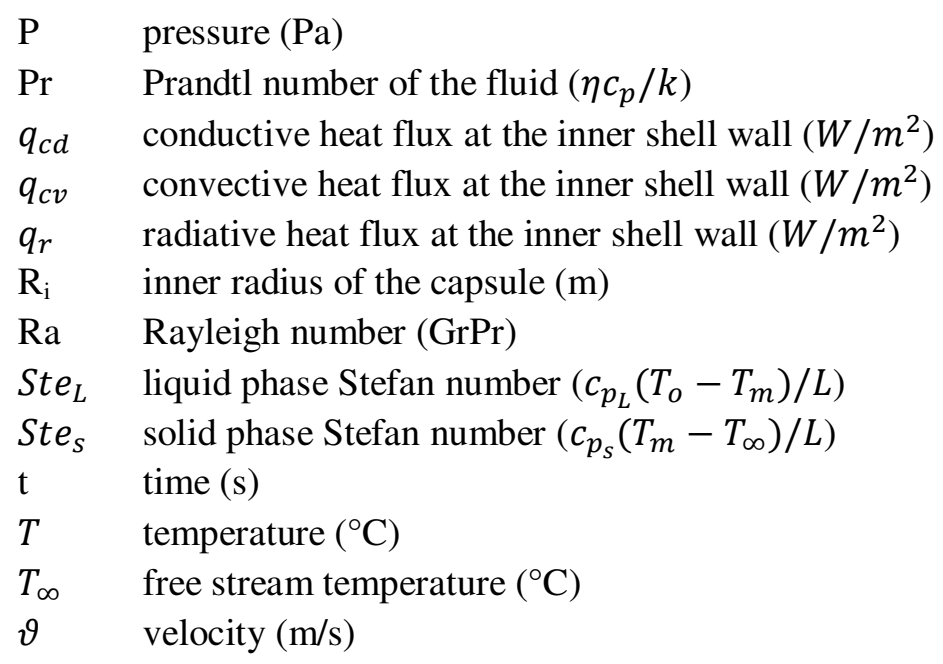

\section{Greek Symbols}

$\alpha \quad$ Thermal diffusivity $\left(\mathrm{m}^{2} / \mathrm{s}\right)$

$\beta \quad$ thermal expansion coefficient $(1 / \mathrm{K})$

$\delta \quad$ shell wall thickness (m)

$\eta \quad$ dynamic viscosity $(\mathrm{kg} / \mathrm{m} \mathrm{s})$

$\kappa \quad$ thermal conductivity $(\mathrm{W} / \mathrm{m} \mathrm{K})$

$\kappa_{a} \quad$ absorption coefficient $\left(\mathrm{m}^{-1}\right)$

$\lambda \quad$ latent heat $(\mathrm{J} / \mathrm{kg})$

$\rho \quad$ density $\left(\mathrm{kg} / \mathrm{m}^{3}\right)$

$\sigma \quad$ Stefan-Boltzmann constant, $\sigma=5.67 \times 10^{-8} \mathrm{~W} / \mathrm{m}^{2} \mathrm{~K}^{4}$

\section{Subscripts}

m melting

o initial

$\mathrm{r} \quad$ radial direction

s solid

w wall

$\theta$ angular direction

$\Psi \quad$ dimensionless radiative flux at the inner shell wall $q_{r} / \sigma\left(T_{m}^{4}-T_{\infty}^{4}\right)$ 


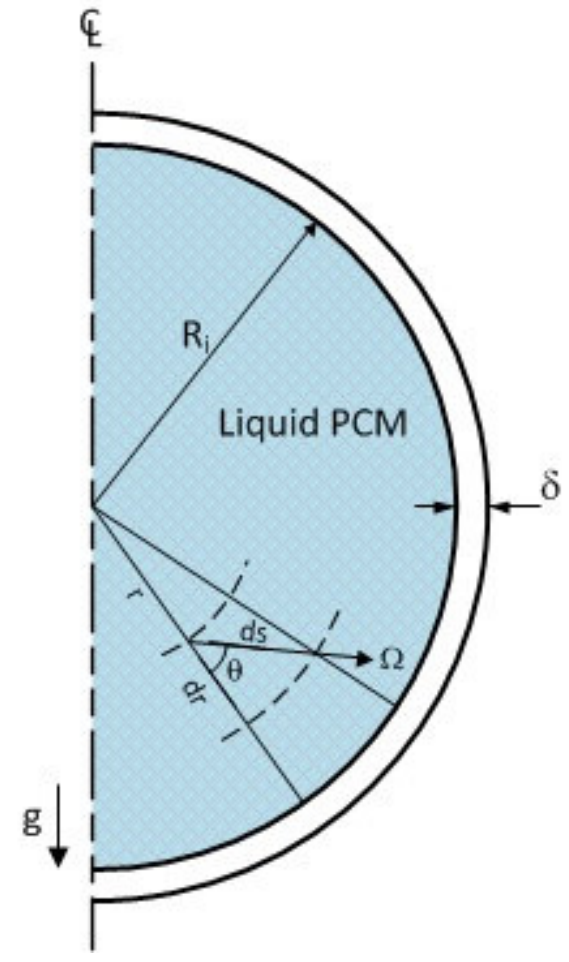

372

Figure 1. Schematic representation of the physical domain.

374 


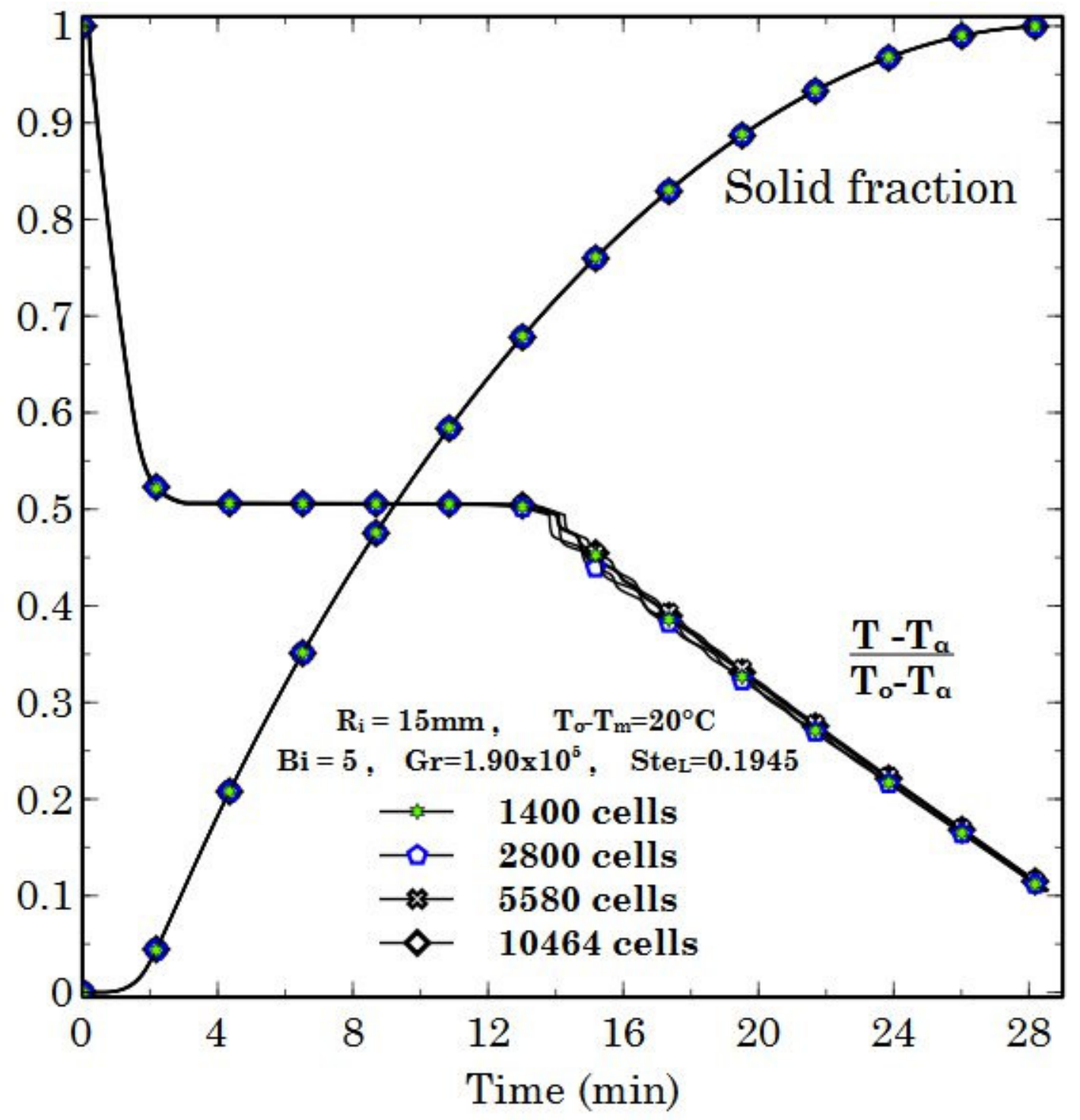




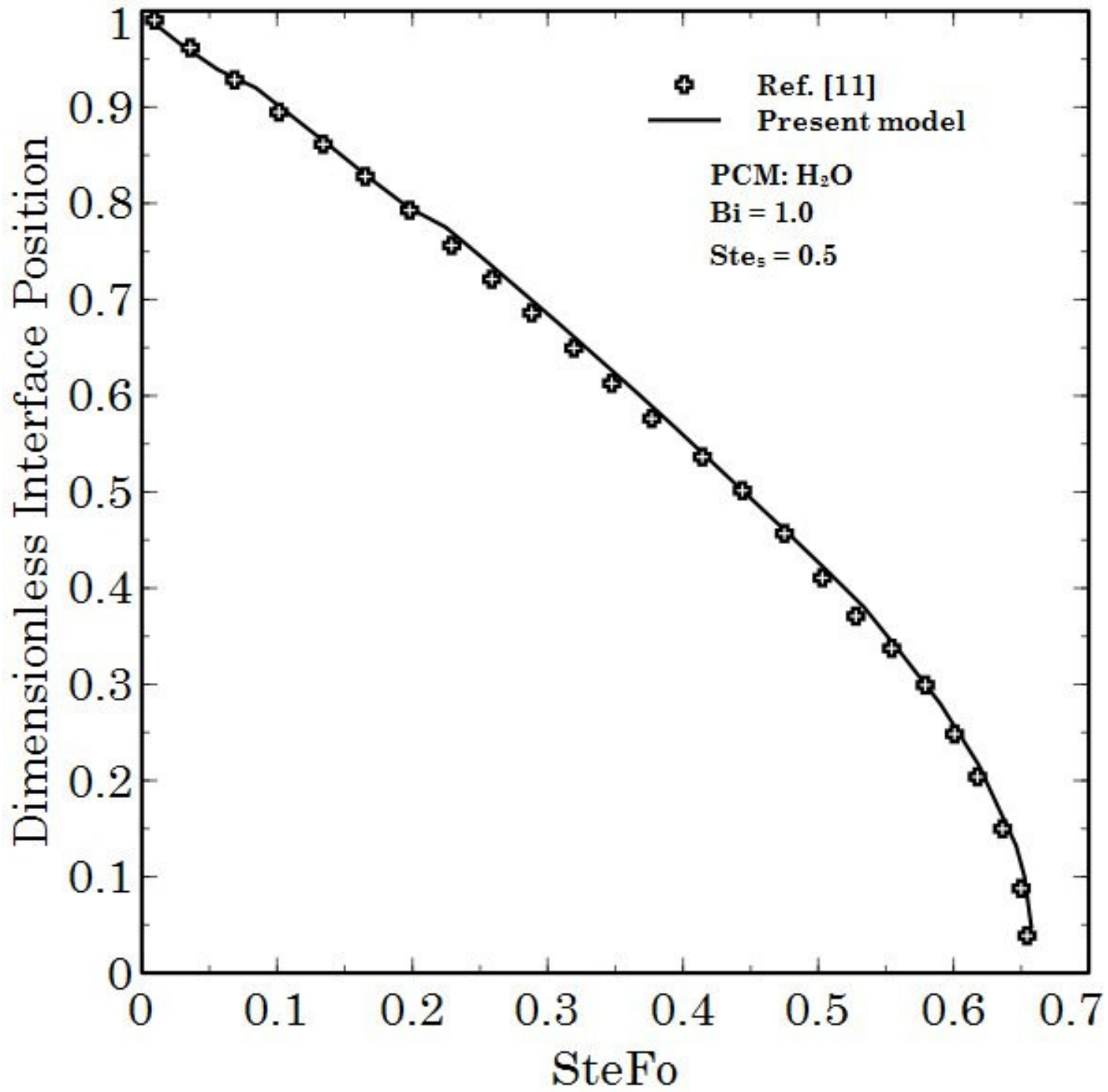




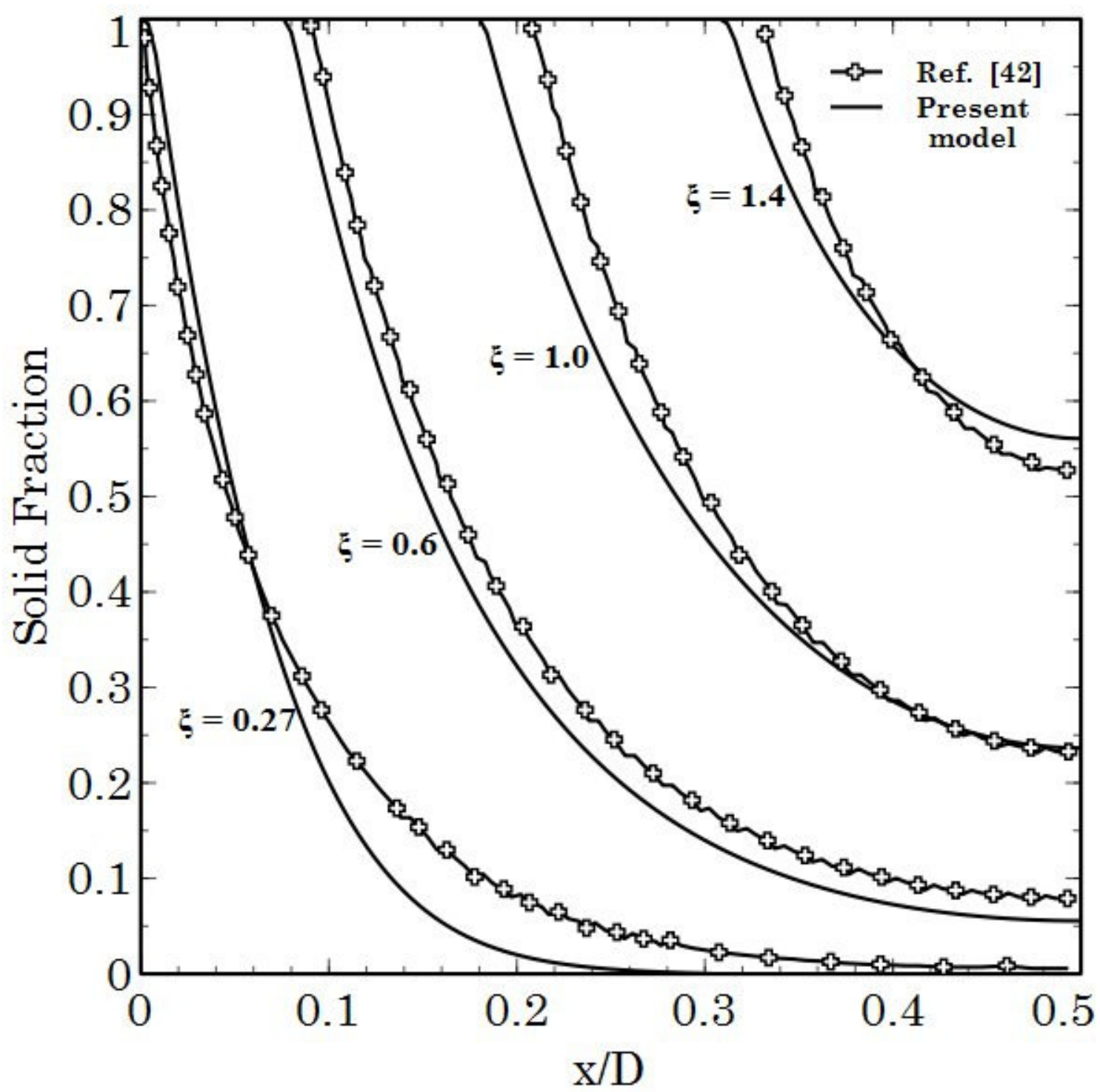




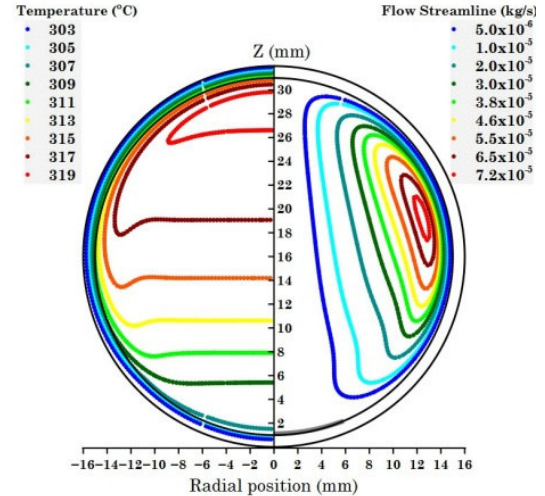

a) $60 \mathrm{~s}$

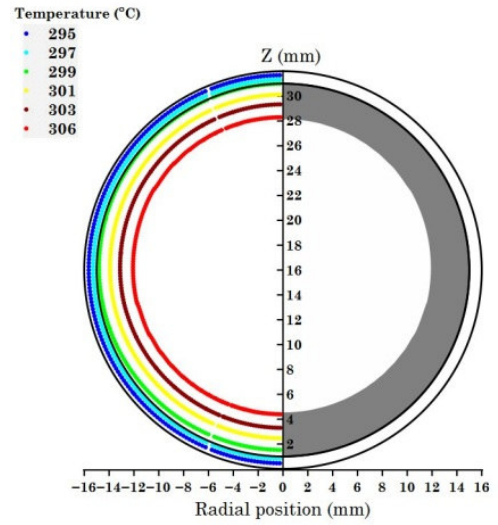

d) $600 \mathrm{~s}$

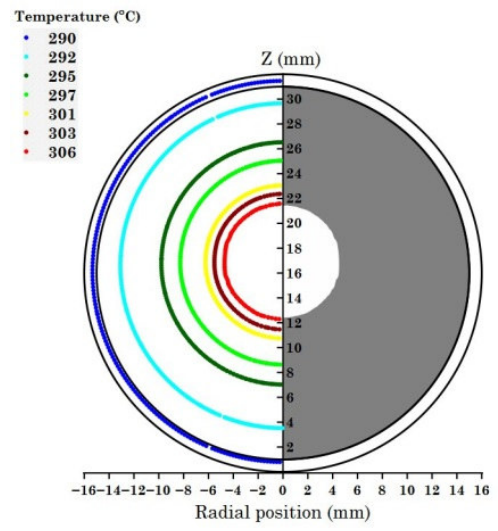

g) $1500 \mathrm{~s}$

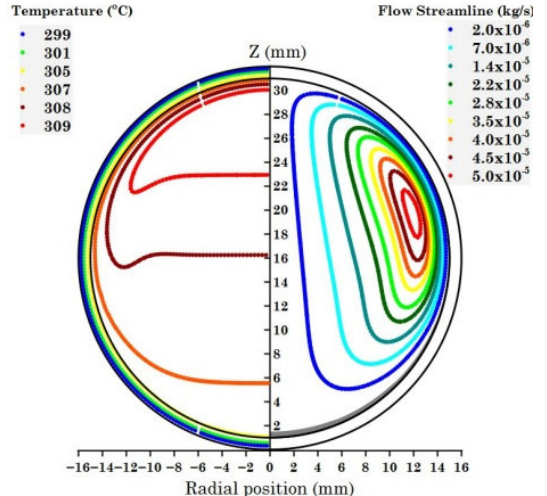

b) $120 \mathrm{~s}$

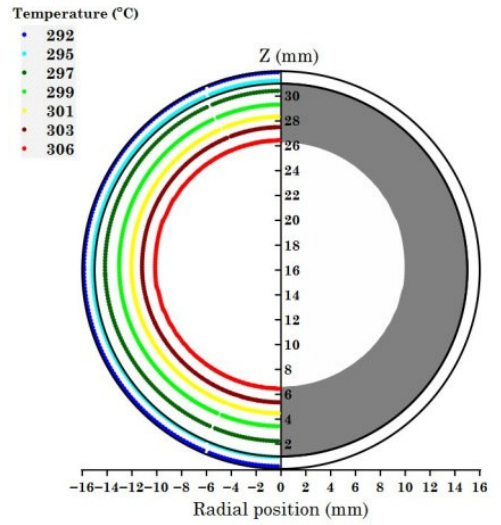

e) $900 \mathrm{~s}$

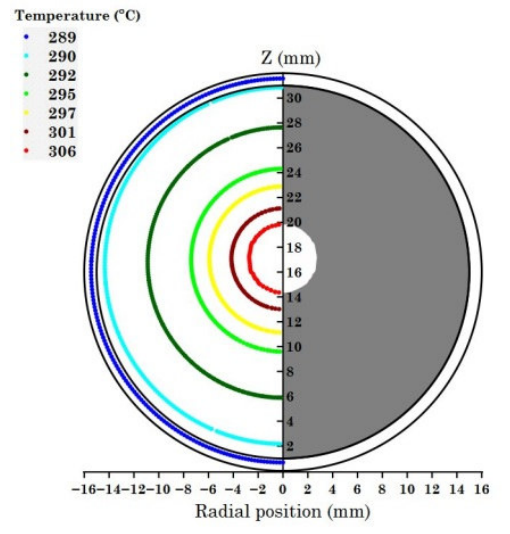

h) $1620 \mathrm{~s}$

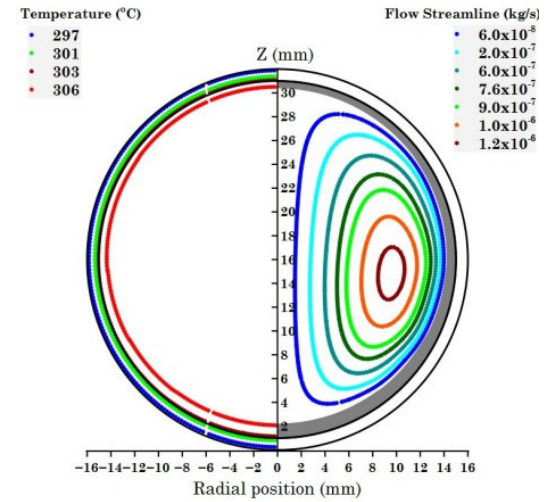

c) $240 \mathrm{~s}$

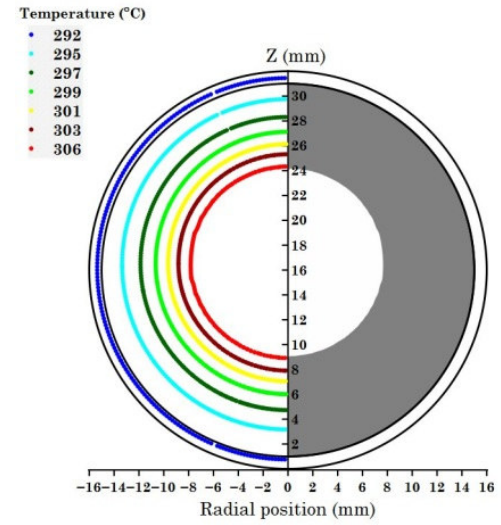

f) $1200 \mathrm{~s}$

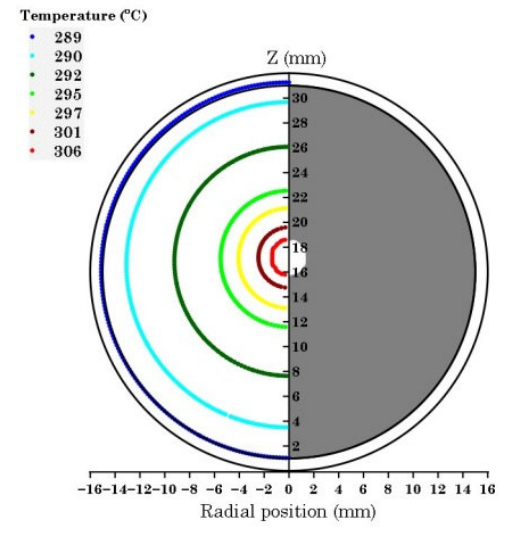

i) $1680 \mathrm{~s}$

Figure 5 Predicted evolution of the solidification process for case5. 


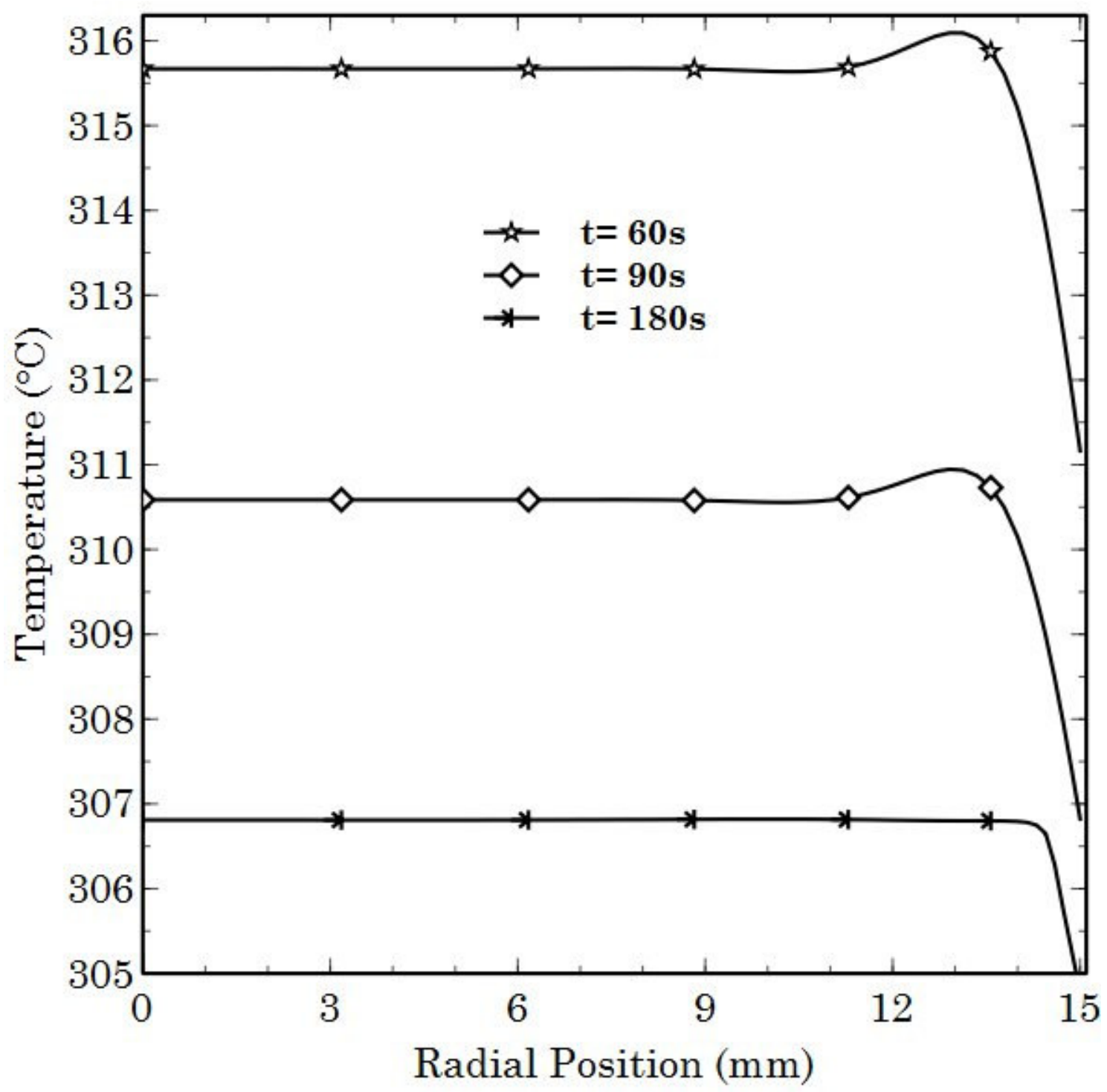




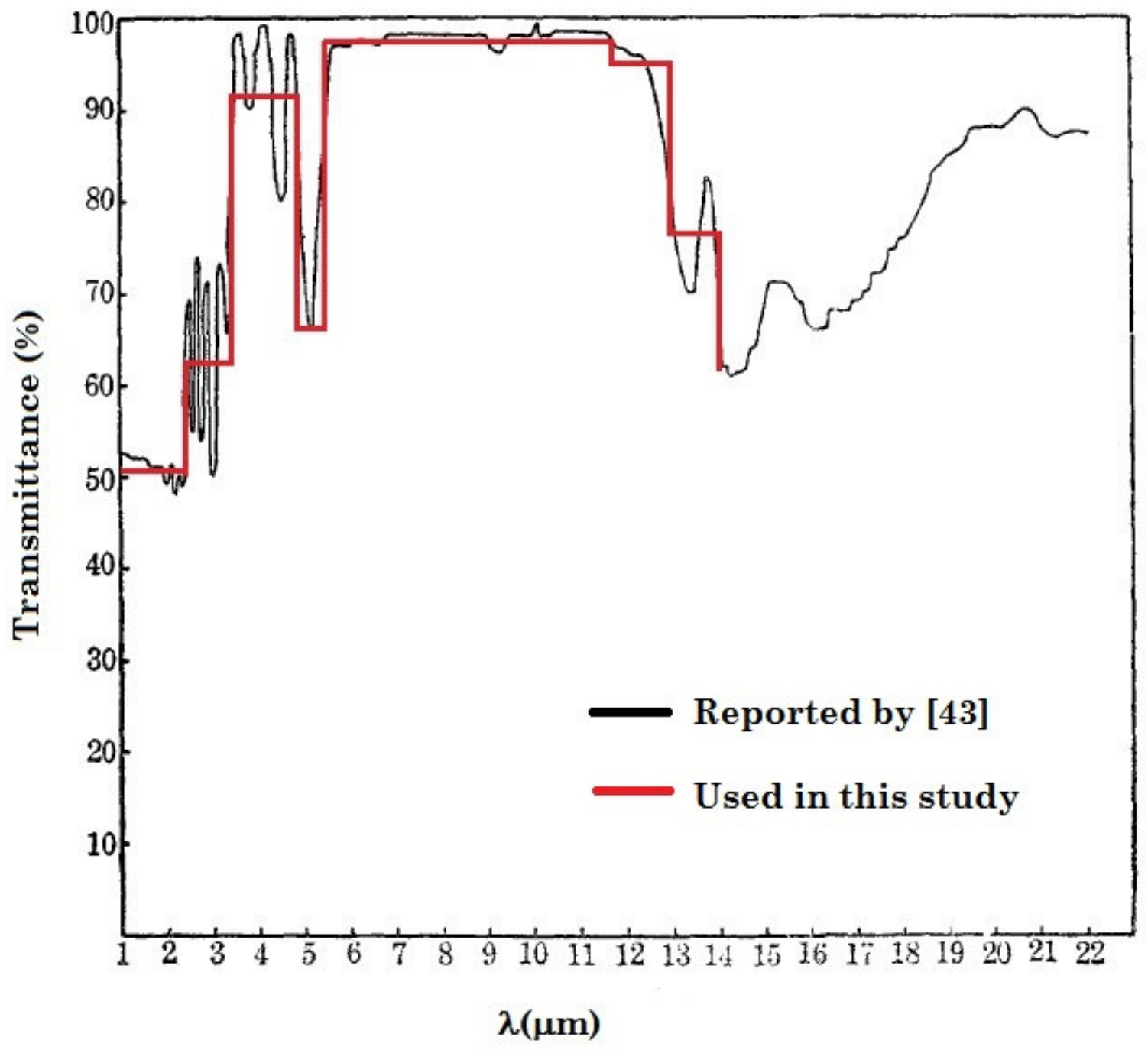

396

Figure 7 Spectral transmittance of Sodium Nitrate. 


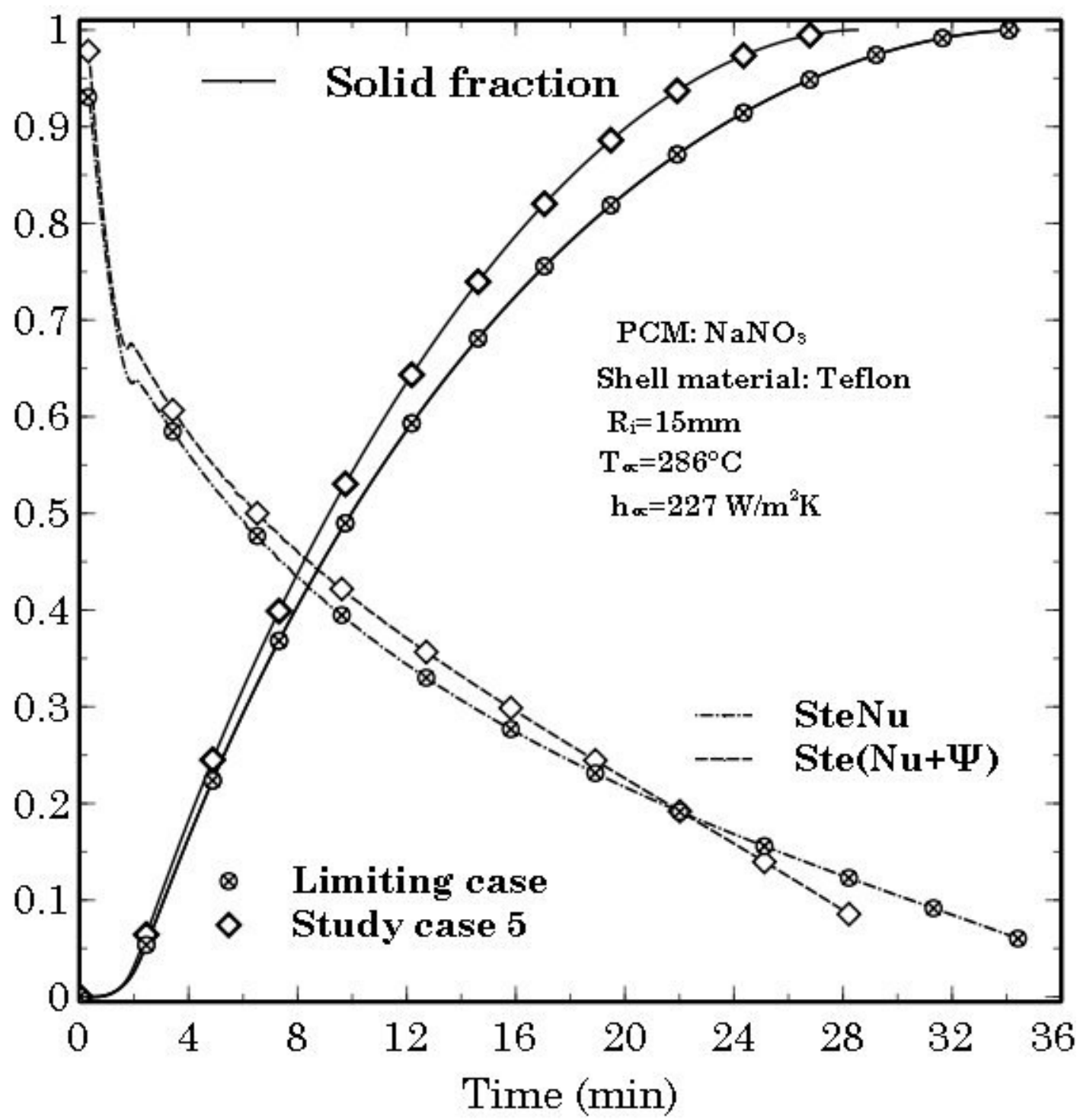




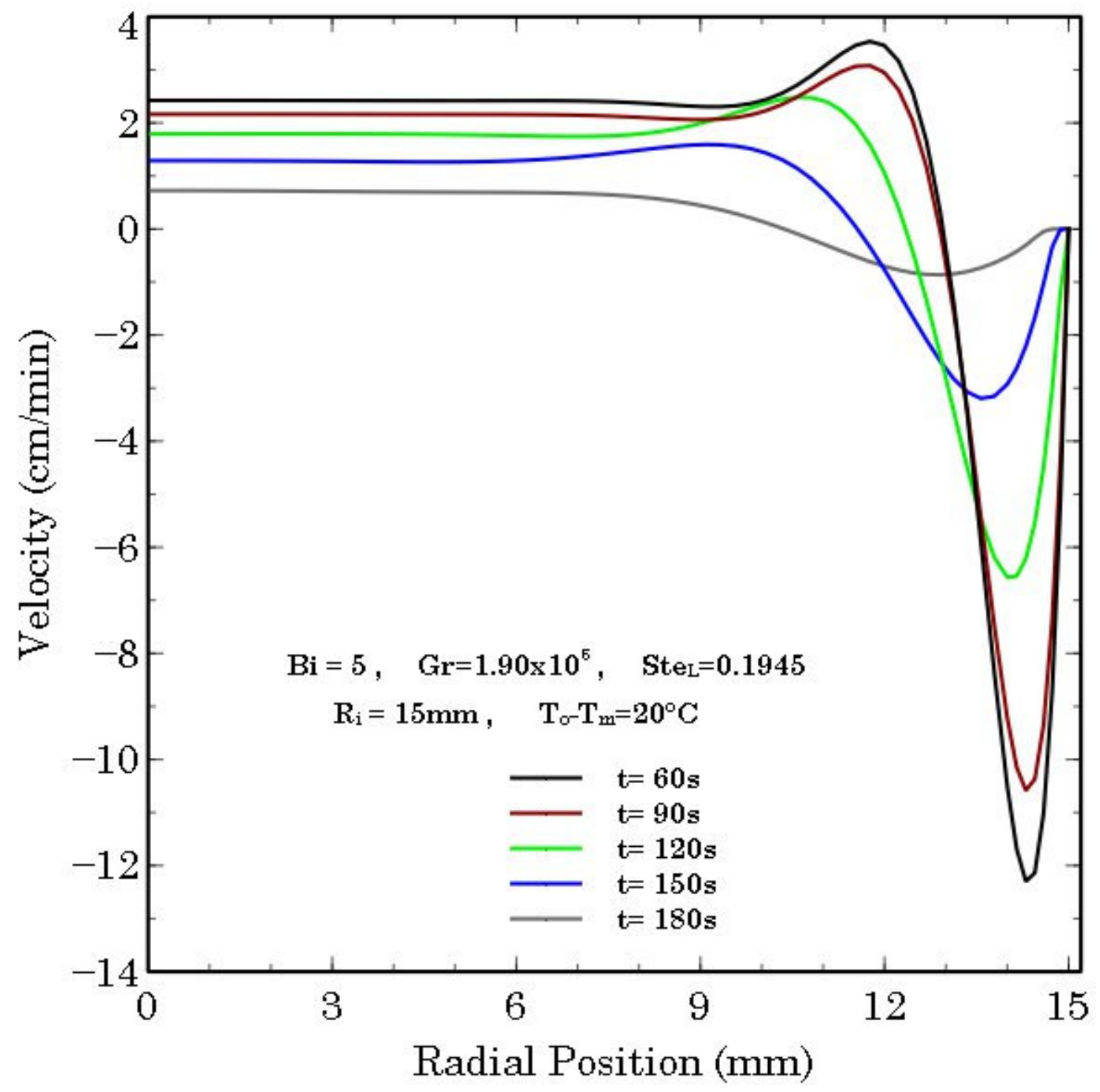




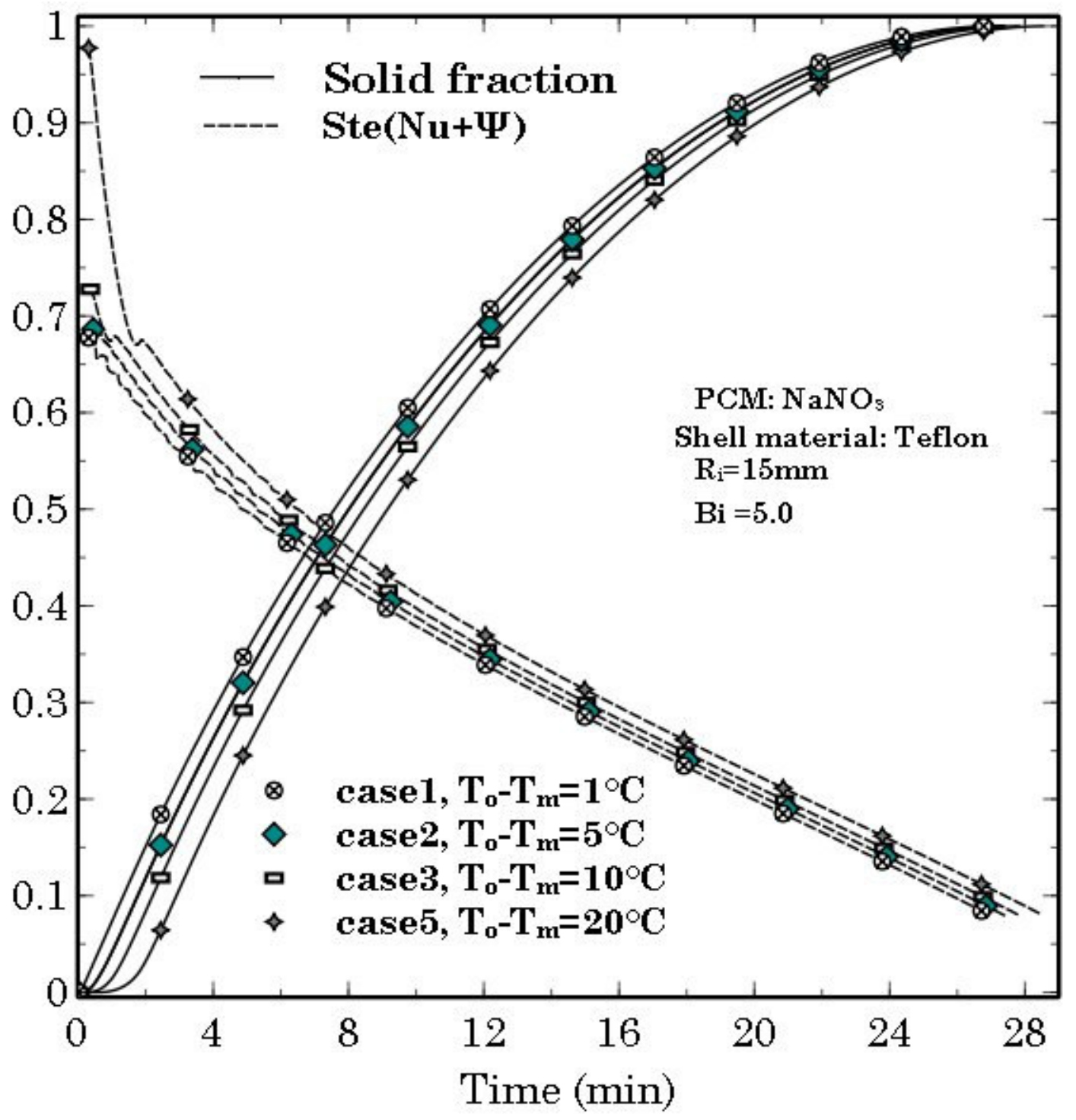




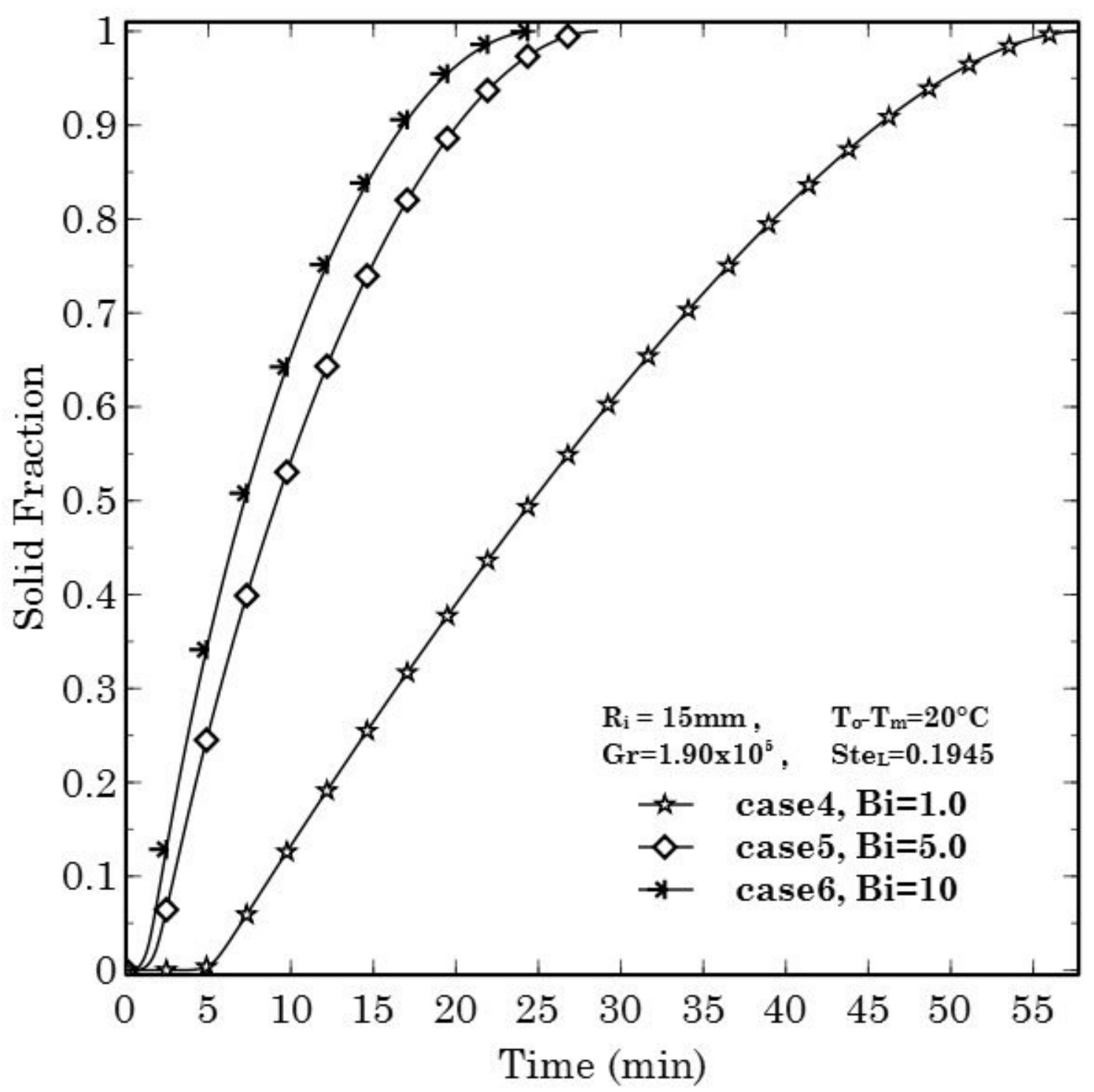

412

413 414

Figure 11 Influence of the Biot number on the solid fraction rate. 


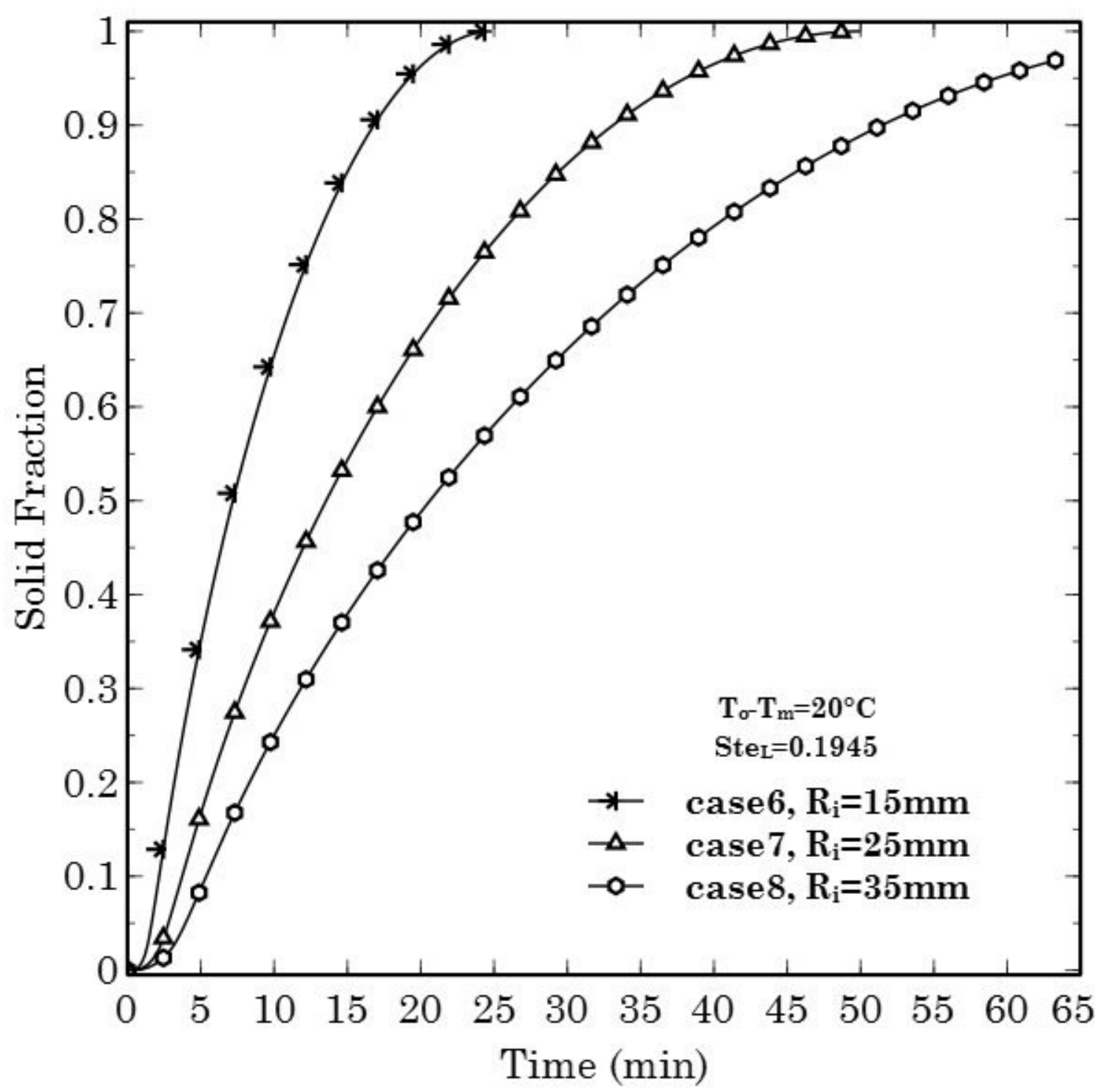

416

Figure 12 Influence of the shell size on the solid fraction rate. 


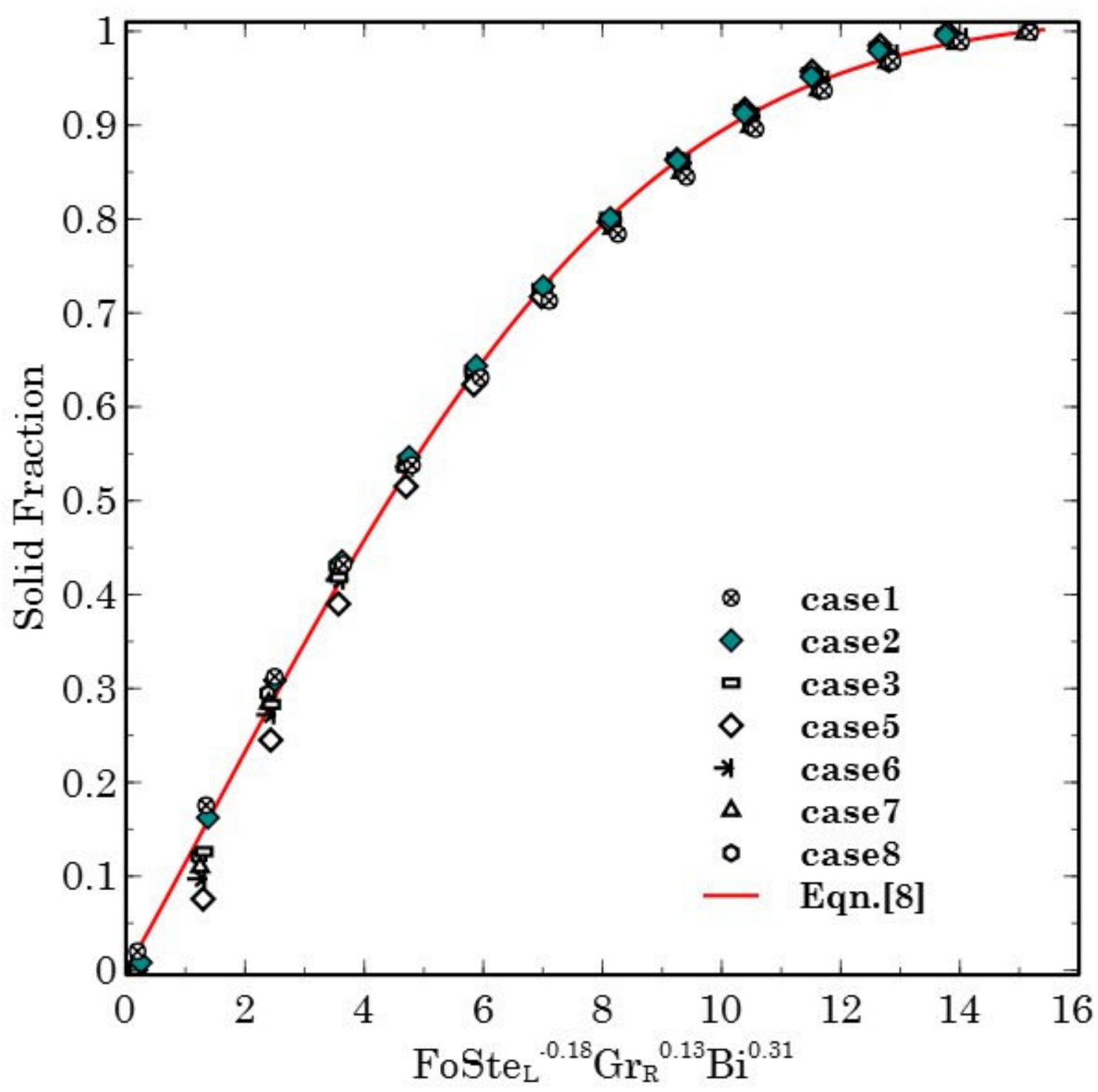

421

Figure 13 Solid fraction as a function of the controlling parameters. 
Table 1 Geometrical and thermal parameters.

\begin{tabular}{cccccc}
\hline \hline case & $R_{i}(m)$ & $T_{o}-T_{m}\left({ }^{\circ} \mathrm{C}\right)$ & $G r_{R}$ & $B i$ & Ste $_{\text {Liq }}$ \\
\hline 1 & 0.015 & 1 & $8.77 \times 10^{3}$ & 5 & 0.0096 \\
2 & 0.015 & 5 & $4.46 \times 10^{4}$ & 5 & 0.048 \\
3 & 0.015 & 10 & $9.09 \times 10^{4}$ & 5 & 0.096 \\
\hline 4 & 0.015 & 20 & $1.90 \times 10^{5}$ & 1 & 0.195 \\
5 & 0.015 & 20 & $1.90 \times 10^{5}$ & 5 & 0.195 \\
6 & 0.015 & 20 & $1.90 \times 10^{5}$ & 10 & 0.195 \\
\hline 7 & 0.025 & 20 & $8.81 \times 10^{5}$ & 16.67 & 0.195 \\
8 & 0.035 & 20 & $2.42 \times 10^{6}$ & 23.34 & 0.195 \\
\hline \hline
\end{tabular}

425

426 


\section{Figure captions.}

428 Figure 1. Schematic representation of the physical domain.

429 Figure 2 Influence of the grid size on the solidification rate and node temperature.

430 Figure 3 Evolution of the solid/liquid interface during single phase Stefan problem.

431 Figure 4 Solid fraction distribution along the slab.

432 Figure 5 Predicted evolution of the solidification process for case5.

433 Figure 6 Temperature distribution along the shell horizontal radius.

434 Figure 7 Spectral transmittance of Sodium Nitrate.

435 Figure 8 Influence of radiative energy transfer on the thermal dynamics of the process.

436 Figure 9 Velocity distribution along the horizontal radius of the shell for study case 5.

437 Figure 10 Influence of the liquid superheating on the thermal dynamics of the process.

438 Figure 11 Influence of the Biot number on the solid fraction rate.

439 Figure 12 Influence of the shell size on the solid fraction rate.

440 Figure 13 Solid fraction as a function of the controlling parameters.

441

\section{Table captions}

443 Table 1 Geometrical and thermal parameters. 